\title{
PPAR $\gamma$ ligands inhibit primary tumor growth and metastasis by inhibiting angiogenesis
}

\author{
Dipak Panigrahy, ${ }^{1}$ Samuel Singer, ${ }^{2,3}$ Lucy Q. Shen, ${ }^{1}$ Catherine E. Butterfield, ${ }^{1}$ \\ Deborah A. Freedman, ${ }^{1}$ Emy J. Chen, ${ }^{1}$ Marsha A. Moses, ${ }^{1}$ Susan Kilroy, ${ }^{1}$ \\ Stefan Duensing, ${ }^{4}$ Christopher Fletcher, ${ }^{5}$ Jonathan A. Fletcher, ${ }^{5}$ Lynn Hlatky, ${ }^{6}$ \\ Philip Hahnfeldt, ${ }^{6}$ Judah Folkman, ${ }^{1}$ and Arja Kaipainen ${ }^{1}$
}

${ }^{1}$ Surgical Research Laboratory, Children's Hospital, Department of Surgery, Harvard Medical School, Boston,
Massachusetts, USA
2Department of Surgery, Brigham and Women's Hospital, Boston, Massachusetts, USA
${ }^{3}$ Department of Surgery, Memorial Sloan-Kettering Cancer Center, New York, New York, USA
${ }^{4}$ Department of Pathology and Harvard Center for Cancer Biology, Harvard Medical School, Boston, Massachusetts, USA
${ }^{5}$ Department of Pathology, Brigham and Women's Hospital, Boston, Massachusetts, USA
${ }^{6}$ Department of Radiation Oncology, Dana-Farber Cancer Institute, Harvard Medical School, Boston, Massachusetts, USA

Several drugs approved for a variety of indications have been shown to exhibit antiangiogenic effects. Our study focuses on the PPAR $\gamma$ ligand rosiglitazone, a compound widely used in the treatment of type 2 diabetes. We demonstrate, for the first time to our knowledge, that PPAR $\gamma$ is highly expressed in tumor endothelium and is activated by rosiglitazone in cultured endothelial cells. Furthermore, we show that rosiglitazone suppresses primary tumor growth and metastasis by both direct and indirect antiangiogenic effects. Rosiglitazone inhibits bovine capillary endothelial cell but not tumor cell proliferation at low doses in vitro and decreases VEGF production by tumor cells. In our in vivo studies, rosiglitazone suppresses angiogenesis in the chick chorioallantoic membrane, in the avascular cornea, and in a variety of primary tumors. These results suggest that PPAR $\gamma$ ligands may be useful in treating angiogenic diseases such as cancer by inhibiting angiogenesis.

J. Clin. Invest. 110:923-932 (2002). doi:10.1172/JCI200215634.

\section{Introduction}

PPAR $\gamma$ is a ligand-activated nuclear receptor that is critical in a variety of biological processes, including adipogenesis, glucose metabolism, and inflammation (1). Genetic knockout of PPAR $\gamma$ in mice interferes with terminal differentiation of trophoblasts and vascularization of the placenta, resulting in death by embryonic day 10 (2). PPAR $\gamma$ functions as an important cellular regulator by inhibiting growth and/or inducing differentiation of adipocytes, monocytes, and cancer cells, including those of breast, colon, and liposarcoma (3-7).

PPAR $\gamma$ is the molecular target of the thiazolidinedione (TZD) class of antidiabetic drugs, which includes

Received for publication April 8, 2002, and accepted in revised form August 6, 2002.

Address correspondence to: Arja Kaipainen, 320 Longwood Avenue, Enders 1050, Boston, Massachusetts 02115, USA. Phone: (617) 355-6167; Fax: (617) 566-6467;

E-mail: arja.kaipainen@tch.harvard.edu.

Dipak Panigrahy and Samuel Singer contributed equally to this work.

Conflict of interest: No conflict of interest has been declared. Nonstandard abbreviations used: thiazolidinedione (TZD); bovine capillary endothelial (BCE); human umbilical vein endothelial cell (HUVEC); Lewis lung carcinoma (LLC); green fluorescent protein (GFP); platelet/endothelial cell adhesion molecule (PECAM); prostatic-specific antigen (PSA); tissue inhibitor of metalloproteinase (TIMP); proliferating cell nuclear antigen (PCNA); chick chorioallantoic membrane (CAM); matrix metalloproteinase (MMP). rosiglitazone and troglitazone. It has been shown that TZDs suppress the growth of several cancer lines, including colon, breast, and prostate, in vitro and in vivo $(6,8,9)$. However, TZDs have minimal to no inhibitory activity on other tumor lines in vitro $(5,8,10$, 11). In fact, high levels of PPAR $\gamma$ in tumor cells do not always correlate with growth inhibition by TZDs in vitro, suggesting that tumor cells can acquire resistance to direct TZD effects $(5,8,12)$. Most importantly, TZDs have antitumor effects in PPAR $\gamma$-deficient embryonic stem cell tumors (12). Together these findings suggested that the antitumor effects of PPAR $\gamma$ ligands may not be mediated entirely through the tumor cell itself.

We hypothesized that the tumor endothelium is a potential target of TZDs, since primary tumor and metastatic growth are dependent on angiogenesis (13). This hypothesis is supported by the observations that PPAR $\gamma$ is expressed in endothelial cells, and that PPAR $\gamma$ ligands can inhibit their proliferation induced by growth factors or cause their apoptosis in vitro (14-17). In addition, TZDs inhibit choroidal neovascularization when administered intraocularly (18). Interestingly, there is recent evidence suggesting that PPAR $\gamma$ is localized in tumor endothelial cells (19). However, the role of PPAR $\gamma$ ligands in tumor angiogenesis has not been studied.

Here we report, for the first time to our knowledge, that PPAR $\gamma$ is expressed at high levels in tumor endothelium and can be activated in endothelial cells by its ligands. We show that PPAR $\gamma$ ligands, such as 
rosiglitazone, inhibit primary tumor growth and metastasis by both direct and indirect antiangiogenic effects. These findings are clinically relevant because rosiglitazone is an orally administered, FDA-approved drug already used by millions for the treatment of type 2 diabetes. Several other angiogenesis inhibitors have also been identified in drugs originally approved for other indications. These include IFN- $\alpha$ (20), thalidomide (21), and celecoxib (22). Results from our studies suggest that PPAR $\gamma$ ligands, such as rosiglitazone, are an addition to this growing class of antiangiogenic drugs for cancer therapy.

\section{Methods}

Cells and reagents. Bovine capillary endothelial (BCE) cells were maintained as described (23). Human umbilical vein endothelial cells (HUVECs; VEC Technologies Inc., Rensselaer, New York, USA) were cultured in microvascular endothelial cell growth medium (EGM-2 MV; Clonetics, Walkersville, Maryland, USA). Human glioblastoma cells (U87), prostate carcinoma (LNCaP), T241 fibrosarcoma, human rhabdomyosarcoma cells (RMS559), and Lewis lung carcinoma (LLC) were cultured in DMEM in heat-inactivated 10\% FBS. LLC-green fluorescent protein (LLC-GFP) cells were kindly provided by T. Boehm (Children's Hospital, Boston, Massachusetts, USA). Human liposarcoma tissue specimens were obtained with patient consent and implanted as pieces $\left(1 \mathrm{~mm}^{3}\right)$ subcutaneously into SCID mice (Massachusetts General Hospital, Boston, Massachusetts, USA) in accordance with institutional guidelines of Brigham and Women's and Children's Hospitals (Boston, Massachusetts, USA). Tumor was excised under sterile conditions, digested with collagenase and DNase (Roche Diagnostics Corporation, Indianapolis, Indiana, USA), and cultured in DMEM/F-12 media with $20 \%$ FBS. Rosiglitazone was kindly provided by GlaxoSmithKline Pharmaceuticals (King of Prussia, Pennsylvania, USA). Troglitazone was kindly provided by Parke-Davis (Ann Arbor, Michigan, USA). WY 14643 was obtained from ChemSyn Laboratories (Lenexa, Kansas, USA).

Preparation of bFGF. Human bFGF (5'-ATGCCCGCCT TGCCCGAGGA TGGCGGCAGC GGCGCCTTCC CGCCCGGCCA CTTCAAGGAC CCCAAGCGGC TGTACTGCAA AAACGGGGGC TTCTTCCTGC GCATCCACCC CGACGGCCGA GTTGACGGGG TCCGGGAGAA GAGCGACCCT CACATCAAGC TACAACTTCA AGCAGAAGAG AGAGGAGTTG TGTCTATCAA AGGAGTGTGT GCTAA-3') was expressed in Escherichia coli. The protein was purified by Fast Performance Liquid Chromatography over a HiTrap Heparin column (Amersham Biosciences Corp., Piscataway, New Jersey, USA). The column was washed with $0.8 \mathrm{M} \mathrm{NaCl}$, and bFGF was eluted with $2 \mathrm{M}$ and $3 \mathrm{M} \mathrm{NaCl}$. Fractions containing pure bFGF as determined by silver stain and Western analysis were combined, concentrated, sterilized, and dialyzed into 20 $\mathrm{mM}$ sodium citrate, $1 \mathrm{mM}$ EDTA, 9\% sucrose, $\mathrm{pH}$ 5.0.

Flow cytometry analysis. A suspension of tumor cells (T241 fibrosarcoma or LLC-GFP) was made by passage of viable tissue through a sieve and treatment with collagenase and DNase. For isolation of skin endothelial cells, murine skin was minced, treated with collagenase and DNase in DMEM, homogenized, and filtered. Cell suspensions were labeled with phycoerythrin-conjugated anti-VEGF receptor-2 and anti-platelet/endothelial cell adhesion molecule (anti-PECAM) antibodies (Pharmingen, San Diego, California). One million positive tumor and skin endothelial cells or GFP-positive LLC cells were collected by FACS.

VEGF, bFGF, and prostatic-specific antigen ELISA. Tumor cells were plated at 15,000 cells per well (six-well plates) and 24 hours later were treated with rosiglitazone or $0.1 \%$ DMSO. Medium containing the drugs was changed on days 3 and 5 . Medium was collected on day 6. VEGF and bFGF in the media were assayed by ELISA (R\&D Systems Inc., Minneapolis, Minnesota, USA). Serum prostatic-specific antigen (PSA) was measured by ELISA (Beckman Coulter Inc., Fullerton, California, USA) as described (24).

Matrix metalloproteinase and tissue inbibitor of metalloproteinase activity. Gelatinase activity in the control media and rosiglitazone-treated cells was determined by substrate gel electrophoresis as previously reported (25). Tissue inhibitor of metalloproteinase (TIMP) activity was analyzed using a modified quantitative $\left[{ }^{14} \mathrm{C}\right]$ collagen film collagenase assay (26).

Western blot analysis. After 16 hours of treatment, protein was extracted from $60 \%$ confluent plates and immunoblotting was performed for PPAR $\gamma$ as described (5). A positive control for PPAR $\gamma$ was obtained from fully differentiated $\mathrm{C} 3 \mathrm{H} / 10 \mathrm{~T} 1 / 2$ cells with adipocyte features, as confirmed with oil red $\mathrm{O}$ staining. Total protein extracts $(10-30 \mu \mathrm{g})$ were analyzed on blots incubated with primary (Calbiochem-Novabiochem Corp., San Diego, California, USA) and secondary antibodies (Amersham Biosciences Corp.). To assay the eIF- $2 \alpha$ phosphorylation state after 30 minutes of treatment, protein was extracted from exponentially growing HUVECs and Western blot analysis was performed as described (12).

Immunobistochemistry. Surgical specimens of tumors and skin were processed according to standard protocol. Sections were microwaved in $10 \mathrm{mM}$ sodium citrate ( $\mathrm{pH}$ 6.0) for PPAR $\gamma, \mathrm{vWF}, \mathrm{MECA}-32$, and proliferating cell nuclear antigen (PCNA) and were treated with 40 $\mu \mathrm{g} / \mathrm{ml}$ proteinase K (Roche Diagnostics Corp.) for 25 minutes at $37^{\circ} \mathrm{C}$ for PECAM. Mouse anti-human PPAR $\gamma($ Santa Cruz Biotechnology Inc., Santa Cruz, California, USA), rabbit anti-human vWF (DAKO Corp., Carpinteria, California, USA), rat anti-mouse PECAM (Pharmingen), MECA-32 rat anti-mouse panendothelial cell antigen (Pharmingen), and mouse anti-human PCNA (DAKO Corp.) were incubated at $4^{\circ} \mathrm{C}$ overnight. PECAM, MECA-32, and PPAR $\gamma$ stainings were amplified using tyramide signal amplification direct and indirect kits (NEN Life Science Products Inc., Boston, Massachusetts, USA). vWF and PCNA were detected with FITC-conjugated secondary antibodies. Rabbit anti- 
human pericentrin (Covance Inc., Richmond, California, USA) was detected with biotinylated secondary antibody after treatment in ice-cold methanol.

Angiogenesis assays. Endothelial cell proliferation was assayed as described (23) with $7.5 \times 10^{3}$ cells per well. For tumor cell proliferation, cells were plated at $5 \times 10^{3}$ cells per well. After 24 hours, cells were starved for 12 hours in $0.5 \%$ FBS. Cells were treated with TZDs dissolved in $0.1 \%$ DMSO. The medium was changed on days 3 and 5 . Cells were counted with a particle counter on days 3 and 7.

Chick chorioallantoic membrane (CAM) assays were performed in three separate experiments as described (23). CAMs were observed 48 hours after implantation of a methylcellulose disc $(10 \mu \mathrm{l})$ with and without rosiglitazone. Corneal neovascularization assays were performed as described (23). After implantation of $80 \mathrm{ng}$ bFGF, TZDs were administered over 6 days by gavage in an aqueous solution of $10 \%$ DMSO in $0.5 \%$ methylcellulose as described (6), and control mice received vehicle. For tumor studies, LLC was injected as described (23). Rhabdomyosarcoma and glioblastoma cells were injected subcutaneously $\left(2 \times 10^{6}\right.$ cells in $\left.0.1 \mathrm{ml} \mathrm{PBS}\right)$. Once tumors were $100-200 \mathrm{~mm}^{3}$, mice were randomized into treatment and vehicle groups. TZDs or vehicle were administered by daily gavage for $14-40$ days. Tumors were measured every $3-5$ days, and volume was calculated as width ${ }^{2} \times$ length $\times 0.52$. Prostate tumor cells (LNCaP) were implanted orthotopically as described (24). Twenty-one days after implantation, mice with serum PSA between 40 and $60 \mathrm{ng} / \mathrm{ml}$ were randomized into treatment and vehicle groups. TZDs or vehicle were administered for 17 days ( $n=6$ mice per group). For metastasis studies, LLC tumors were resected 14 days after implantation as described (23). After LLC resection, mice were treated with TZDs or vehicle for 17 days when control mice became terminally ill. On the last day of treatment, the statistical difference between treatment and control groups was determined by Student's $t$ test. A $P$ value less than 0.05 was accepted as significant.

Computer-enhanced imaging of tumors. Histological sections of tumors were analyzed for vessel density by computerized densitometric imaging (Quantimet 570; Leica Inc., Deerfield, Illinois, USA; JVC 3-CCD camera; Victor Co., Tokyo, Japan). The degree of vascularization was quantified over the entire tumor section and expressed as a ratio of vessel area (CD31) to tumor area. Histograms represent the distribution of ratios compiled for 124-314 microscope fields at $\times 125$ magnification.

\section{Results}

PPAR $\gamma$ is highly expressed in tumor endothelium in vivo and proliferating endothelial cells in vitro

We first analyzed the expression pattern of PPAR $\gamma$ in the endothelium of normal skin and of various tumors by immunofluorescent double staining for PPAR $\gamma$ and vWF, an endothelial marker. PPAR $\gamma$ was expressed in endothelial and mural cells of normal skin (Figure 1a), in tumor cells, and in the endothelium of all tumors studied including human glioblastoma tissue (Figure 1b). PPAR $\gamma$ localization in the nucleus was confirmed by Hoechst staining.

Although we screened 11 tumor cell lines for a PPAR $\gamma$ negative tumor, all tumor cell lines expressed PPAR $\gamma$ protein, at varying levels (Figure 1c). To confirm expression of PPAR $\gamma$ in tumor endothelial cells, we separated endothelial cells from a T241 fibrosarcoma cell suspension.
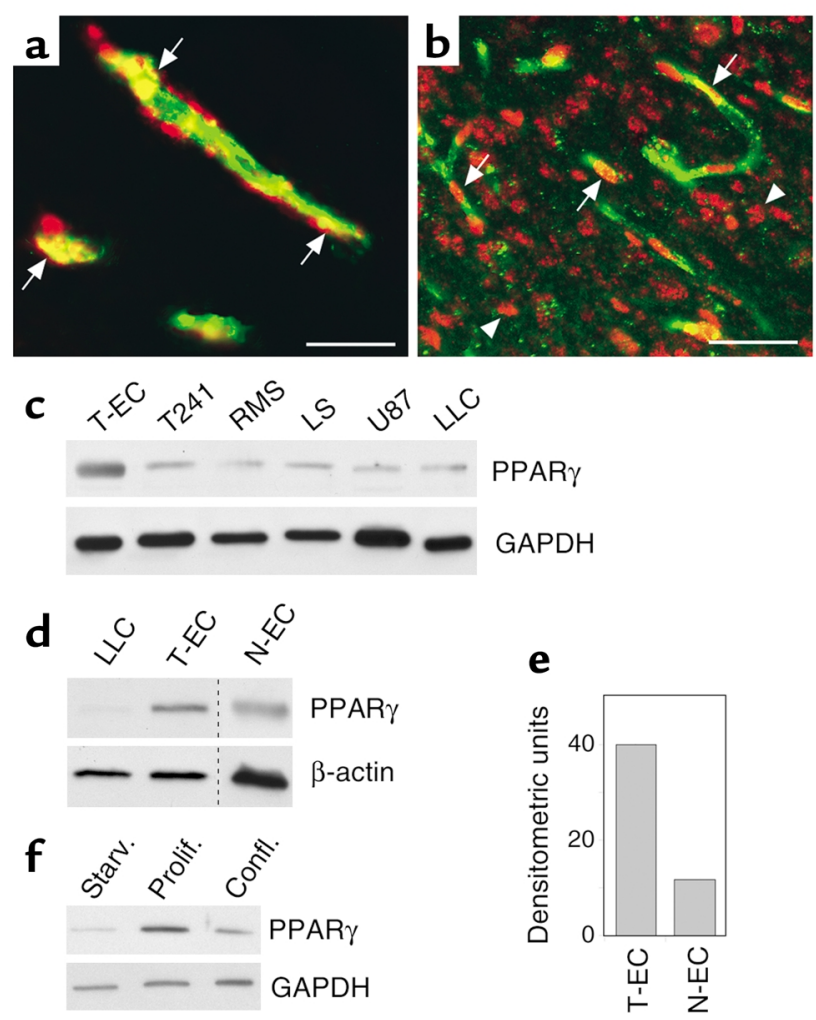

\section{Figure 1}

PPAR $\gamma$ is expressed in endothelium of normal and neoplastic tissues. (a) Immunofluorescent double staining for VWF and PPAR $\gamma$ demonstrates PPAR $\gamma$ expression in endothelium of normal skin. vWF-stained endothelial cells are shown in green, and PPAR $\gamma$-positive cells are red. PPAR $\gamma$-positive nuclei inside the endothelium appear yellow (arrows). Red cells are PPAR $\gamma$-expressing mural cells. Scale bar, $20 \mu \mathrm{m}$. (b) In human glioblastoma tissue, PPAR $\gamma$ (red) is expressed in both tumor (arrowheads) and endothelial cells. Colocalization of red and green fluorescence indicates PPAR $\gamma$ in blood vessels (arrows). Scale bar, 20 $\mu \mathrm{m}$. (c) Western blot analysis of PPAR $\gamma$ expression of cell lysates from isolated tumor endothelial cells from T241 fibrosarcoma (T-EC), and from cultured tumor cells including T241 fibrosarcoma (T241), rhabdomyosarcoma (RMS), liposarcoma (LS), glioblastoma (U87), and LLC. (d) Western blot analysis of PPAR $\gamma$ expression from isolated LLC tumor cells (LLC) and corresponding endothelial cells from LLC-GFP tumor (T-EC) and skin endothelial cells ( $N$-EC). Levels of $\beta$-actin demonstrate protein loading. (e) Quantitation of autoradiographic signals. Values represent arbitrary densitometric units normalized for GAPDH signals. T-EC, tumor endothelium; N-EC, normal (skin) endothelium. (f) Western blot analysis of PPAR $\gamma$ protein showing higher expression in proliferating than in quiescent endothelium. Starv., starvation; Prolif., proliferation; Confl., confluent (contact inhibition). GAPDH levels are shown to demonstrate equal loading of protein in each lane. 


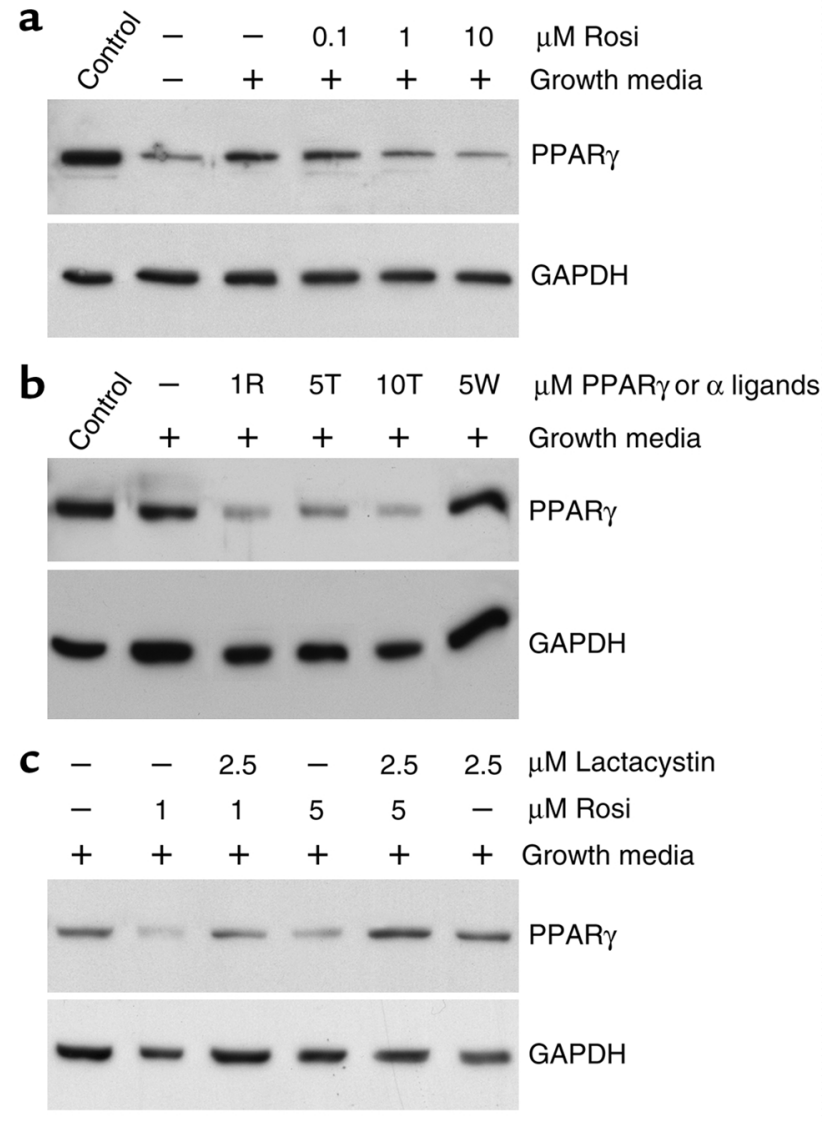

Figure 2

TZDs activate endothelial PPAR $\gamma$ in a lactacystin-reversible manner. (a) Western blot analysis of PPAR $\gamma$ protein in proliferating HUVECs. PPAR $\gamma$ protein levels decrease in a dose-dependent manner with rosiglitazone (Rosi). Control, $\mathrm{C} 3 \mathrm{H} / 10 \mathrm{~T} 1 / 2$ (fully differentiated $\mathrm{C} 3 \mathrm{H}$ cells), a positive control for PPAR $\gamma ;-$, HUVECs cultured in starvation media; +, HUVECs cultured in stimulation (growth) media. (b) PPAR $\gamma$ protein decreases with $5 \mu \mathrm{M}(5 \mathrm{~T})$ and $10 \mu \mathrm{M}(10 \mathrm{~T})$ troglitazone. In contrast, PPAR $\alpha$ activator WY 14643 at $5 \mu \mathrm{M}(5 \mathrm{~W})$ has no effect. 1R, $1 \mu \mathrm{M}$ concentration of rosiglitazone. (c) PPAR $\gamma$ activation can be reversed by coadministration of $2.5 \mu \mathrm{M}$ lactacystin. HUVECs treated with both rosiglitazone and lactacystin (lanes 3 and 5) show PPAR $\gamma$ expression comparable to that of HUVECs treated with stimulation medium alone (lane 1). Lactacystin alone has no effect on basal PPAR $\gamma$ levels (lane 6).

Interestingly, PPAR $\gamma$ protein was highly expressed in isolated tumor endothelial cells (Figure 1c). Furthermore, we observed higher expression levels of PPAR $\gamma$ in endothelial cells than in tumor cells when both cell types were isolated from LLC tumors expressing GFP (Figure 1d). In addition, we compared PPAR $\gamma$ expression in proliferating endothelium (tumor) and quiescent endothelium (skin) (Figure 1d). There was a $71 \%$ higher expression of PPAR $\gamma$ in tumor endothelium (Figure 1e).

To determine whether endothelial cell growth conditions affect PPAR $\gamma$ expression, we performed immunoblotting on whole-cell lysates of HUVECs under proliferating, contact-inhibited, or starvation conditions. Cells were grown in the presence of $2.5 \% \mathrm{FBS}$, bFGF, VEGF, EGF, and IGF-1 in growth-stimulatory condi- tions and in $0.5 \%$ FBS and no growth factors in starvation conditions. In both conditions cells were $60 \%$ confluent. We determined that PPAR $\gamma$ protein levels were higher in cells cultured in growth-stimulating conditions compared with those in 6-day $100 \%$ confluent cultures (contact-inhibited endothelium) or those in starvation medium (Figure 1f). These data demonstrate that PPAR $\gamma$ expression is higher in proliferating endothelium than in quiescent endothelium.

TZDs induce PPAR $\gamma$ activation

in endothelial cells in vitro

Activation of PPAR $\gamma$ by its ligands results in proteolytic degradation of the receptor itself (27). To demonstrate PPAR $\gamma$ activation in endothelial cells, we performed a kinetic study of PPAR $\gamma$ levels in endothelial cells after TZD treatment. After 17 hours of rosiglitazone treatment, PPAR $\gamma$ protein levels decreased in growth factor-stimulated cells in growth media in a dose-dependent manner, indicating PPAR $\gamma$ activation (Figure 2a). Troglitazone similarly reduced PPAR $\gamma$ level in a dose-dependent manner, while PPAR $\alpha$ ligand (WY 14643) had no effect. These data demonstrate that PPAR $\gamma$ downregulation results specifically from PPAR $\gamma$ activation (Figure $2 \mathrm{~b}$ ).

It has been shown that the ubiquitin-proteosome pathway regulates the stability of PPAR $\gamma$ protein in adipocytes (27). Therefore, we asked whether PPAR $\gamma$ is regulated in endothelial cells in a similar manner. In the presence of $2.5 \mu \mathrm{M}$ lactacystin, a proteosome inhibitor, rosiglitazone failed to trigger PPAR $\gamma$ degradation (Figure 2c). The fact that lactacystin can fully reverse the effect of rosiglitazone suggests that rosiglitazone may induce PPAR $\gamma$ degradation by proteosomes in endothelial cells.

TZDs have direct and indirect antiangiogenic effects in vitro

Direct effects. To show the effects of endothelial PPAR $\gamma$ activation on cell proliferation, we used BCE cells and induced their proliferation with bFGF, a potent mitogen for BCE cells, in a standard proliferation assay as reported $(23,28,29)$. Rosiglitazone inhibited bFGFinduced proliferation of BCE cells in a dose-dependent manner, with maximal inhibition of approximately $94 \%$ after a 72-hour incubation period at $1 \mu \mathrm{M}$ (Figure 3a) and about $69-74 \%$ after 7 days of treatment at $0.01-0.1$ $\mu \mathrm{M}$ (Figure 3b). Troglitazone had a similar effect on BCE cell proliferation (data not shown).

In contrast, rosiglitazone did not significantly inhibit proliferation of rhabdomyosarcoma, glioblastoma, LLC, and liposarcoma cells after a 72-hour incubation period, even at doses tenfold $(10 \mu \mathrm{M})$ the concentration used to inhibit endothelial cells (Figure 3a). Even after 7 days of treatment, rosiglitazone only resulted in maximal inhibition of $26 \%$ of liposarcoma and rhabdomyosarcoma cell growth at all concentrations tested, while glioblastoma and LLC were relatively resistant to the antiproliferative effects of rosiglitazone (Figure 3b). 
Because TZDs activate PPAR $\alpha$ and PPAR $\delta$ receptors at greater than $10 \mu \mathrm{M}(30)$, we used concentrations of $10 \mu \mathrm{M}$ and less.

The inhibitory effect of TZDs has recently been suggested to be independent of PPAR $\gamma$ and mediated by inhibition of translation initiation (12). To determine whether endothelial cell growth was inhibited in such a manner, we studied the phosphorylation of translation initiation factor eIF- $2 \alpha$ in response to rosiglitazone at the same concentration range as we used for proliferation studies. We found that eIF- $2 \alpha$ was partially phosphorylated when HUVECs were treated with $5 \mu \mathrm{M}$ rosiglitazone and fully phosphorylated with $10 \mu \mathrm{M}$ rosiglitazone (Figure 3c). However, at $0.01-1 \mu \mathrm{M}$ rosiglitazone, a concentration that was effective in inhibiting endothelial cell growth, there was no effect on eIF- $2 \alpha$ phosphorylation (Figure $3 \mathrm{c}$ ). These results suggest that the growth inhibitory effect of rosiglitazone on endothelial cells is most likely due to PPAR $\gamma$ activation and not to inhibition of translational initiation.

Indirect effects. To determine whether rosiglitazone could indirectly inhibit angiogenesis by downregulating tumor-secreted growth factors, we measured bFGF and VEGF levels in tumor-conditioned media. All tumor lines expressed negligible levels of bFGF, but two tumors, glioblastoma and LLC, showed substantial production of VEGF: $20,000 \mathrm{pg} / 10^{6}$ cells and 938 $\mathrm{pg} / 10^{6}$ cells, respectively. Rosiglitazone at $10 \mu \mathrm{M}$ inhibited VEGF secretion in LLC cells by $56 \%$ and at 1 $\mu \mathrm{M}$ inhibited VEGF secretion in U87 cells by $42 \%$ (Figure $3 \mathrm{~d}$ ). The inhibitory effect of TZDs on VEGF secretion by tumor cells suggests an indirect effect on the endothelium in vivo via tumor cell production of the angiogenic mitogen VEGF.

\section{PPAR $\gamma$ ligands inhibit CAM angiogenesis and bFGF-induced corneal neovascularization}

To test the ability of PPAR $\gamma$ ligands to inhibit in vivo angiogenesis, we examined the effect of rosiglitazone on the de novo growth of blood vessels on the CAMs of 6-day-old chick embryos as reported $(23,28)$. An advantage of the CAM assay is that it provides a macrophage-free environment until day 8 (31). After 48-hour treatment with rosiglitazone, CAM angiogenesis was inhibited in a dose-dependent manner: $0.1 \mu \mathrm{g}$ had no effect; $1 \mu \mathrm{g}$ resulted in a slight inhibitory effect with an avascular zone of 1-2 mm; $2.5 \mu \mathrm{g}$ caused a 3 -mm avascular zone around the disc (Figure 4b).

To optimize the antiangiogenic doses of TZDs for daily administration in mice, we implanted $80 \mathrm{ng}$
bFGF pellets into the corneas of mice to stimulate corneal neovascularization over 6 days, as reported (23, 28, 29) (Figure 4c). Systemic oral administration of rosiglitazone significantly inhibited angiogenesis in a dose-dependent fashion: $50 \mathrm{mg} / \mathrm{kg} / \mathrm{d}$ resulted in $39 \%$ inhibition (Figure 4d); $100 \mathrm{mg} / \mathrm{kg} / \mathrm{d}, 60 \%$ (Figure 4e); $200 \mathrm{mg} / \mathrm{kg} / \mathrm{d}, 61 \%$. Interestingly, much higher doses $(400 \mathrm{mg} / \mathrm{kg} / \mathrm{d})$ caused only $18 \%$ inhibition of vessel growth (Figure 4f). Administration of rosiglitazone twice per day also significantly inhibited angiogenesis at the dose of $5 \mathrm{mg} / \mathrm{kg}$ twice a day (36\%). Daily administration exhibited a biphasic response with an optimal effect at $100-200 \mathrm{mg} / \mathrm{kg} / \mathrm{d}$ (Figure 4g). Systemic oral troglitazone also inhibited bFGF-induced corneal angiogenesis (data not shown).

Systemic therapy with PPAR $\gamma$ ligands inhibits a variety of tumors by angiogenesis inhibition

In order to test whether antiangiogenic therapy with PPAR $\gamma$ ligands could suppress the growth of primary tumors, we treated established subcutaneous tumors of 100-200 $\mathrm{mm}^{3}$ volume grown in syngeneic mice as reported (29). Rosiglitazone at $100 \mathrm{mg} / \mathrm{kg} / \mathrm{d}$ inhibited the growth of glioblastoma, LLC, rhabdomyosarcoma, and liposarcoma by $71 \%, 60 \%, 82 \%$, and $69 \%$ respectively (Figure 5, a-d). There was no evidence of weight loss or other drug-related toxicity in any of the mice. To support our hypothesis that PPAR $\gamma$ ligands can inhibit primary tumor growth by inhibiting angiogenesis, we
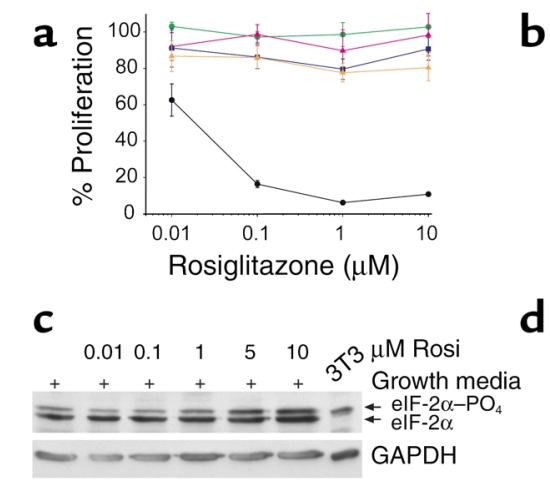

Figure 3

TZDs have direct and indirect antiangiogenic effects. (a) Percent proliferation of BCE cells is determined by comparing cells exposed to an angiogenic stimulus (bFGF) with those exposed to bFGF and rosiglitazone, relative to unstimulated cells (low serum), in a 72-hour proliferation assay. Percent proliferation of tumor cells is determined by comparing cells exposed to $10 \%$ FBS with those exposed to $10 \%$ FBS and rosiglitazone, relative to starvation conditions $(0.5 \% \mathrm{FBS})$. In both cases, percent proliferation $=100 \times\left(\right.$ cell $_{\mathrm{s}_{\text {stimulated }}+\mathrm{TZD}^{-}}$ cells low serum $) /\left(\right.$ cell $_{s_{\text {stimulated }}}$ - cell Slow serum $\left._{\text {loch }}\right)$. Each point represents the mean \pm SD for three wells. Representative experiments of three separate assays are shown. The difference in inhibition between day 3 and day 7 is a result of increased endothelial cell death on day 7

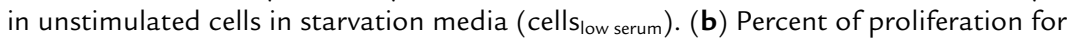
endothelial and tumor cells treated for 7 days with rosiglitazone. (c) Levels of the phosphorylated form of elF- $2 \alpha$ protein upon treatment with rosiglitazone in HUVECs. NIH3T3 fibroblasts (3T3) serve as a positive control. (d) VEGF levels (expressed as percent decrease) in U87 and LLC cells after 6 days of treatment with rosiglitazone. 

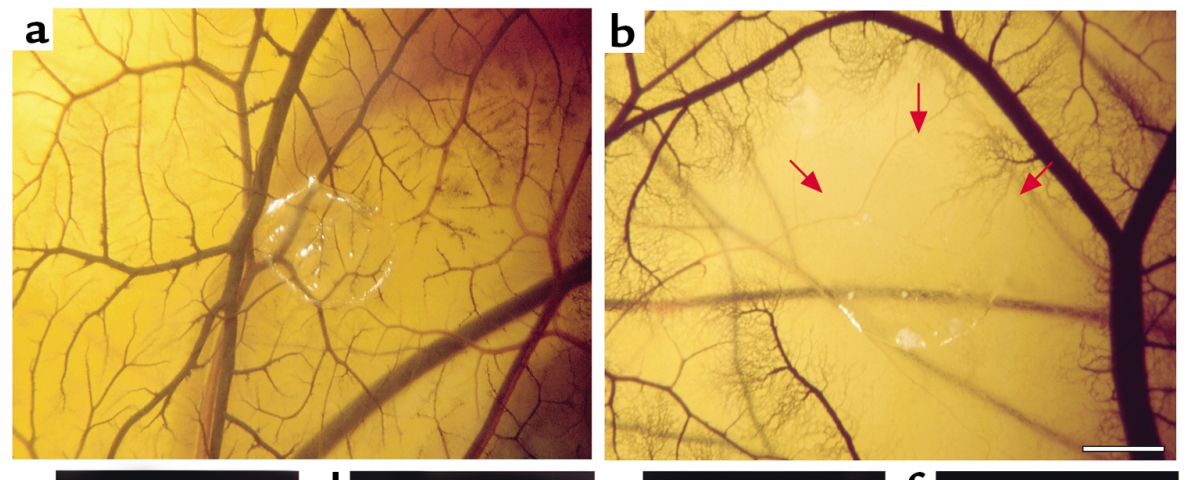

\section{Figure 4}

PPAR $\gamma$ ligands inhibit in vivo angiogenesis in the CAM and bFGF-induced corneal neovascularization. (a) A normal CAM implanted with a methylcellulose disk. (b) After a 48-hour exposure to rosiglitazone $(2.5 \mu \mathrm{g})$, avascular zones free of capillaries and small vessels were observed around the disc (arrows point to a $3-\mathrm{mm}$ avascular zone). Scale bar, $22.5 \mathrm{~mm}$. (c) bFGFinduced neovascularization in control cornea on day 6. (d-f) Systemic treatment with rosiglitazone at $50 \mathrm{mg} / \mathrm{kg} / \mathrm{d}$ (d), $100 \mathrm{mg} / \mathrm{kg} / \mathrm{d}$ (e), and $400 \mathrm{mg} / \mathrm{kg} / \mathrm{d}$ (f). (g) Area of inhibition (percent) by various doses of daily and twice-daily rosiglitazone administration. Inhibition was determined on day 6 by the formula $0.2 \times \pi \times$ neovessel length $\times$ clock hours of neovessels $(n=6$ eyes per group; experiment was performed three times). Lines are fitted to the data assuming a Gaussian distribution.

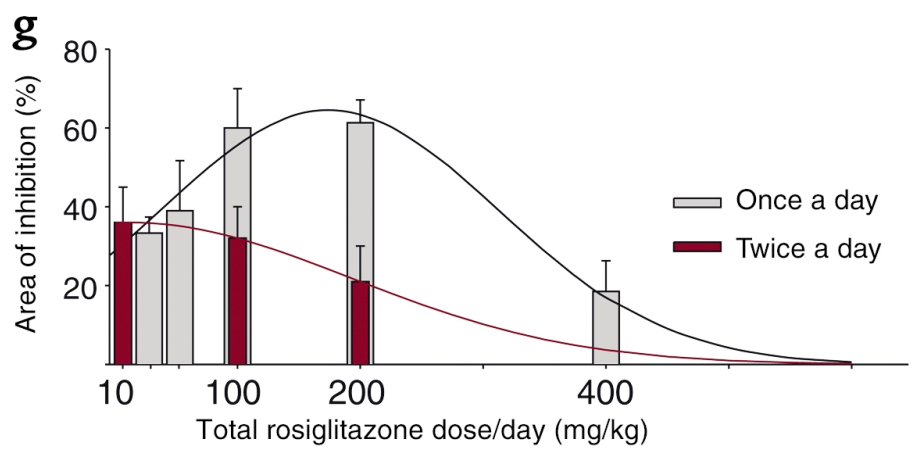

measured microvessel density in treated and control tumors. A decrease in microvessel density during treatment with an angiogenesis inhibitor suggests that the agent is effective (32). However, because microvessel density has limitations as a surrogate marker for antiangiogenic activity in vivo (33), we also measured endothelial cell proliferation in treated and control tumors.

Rosiglitazone treatment reduced vessel density relative to that in the control tumors (Figure 6, a-d). In addition, rosiglitazone-treated tumors showed no signs of cytologic differentiation in vivo (Figure 6, a-d, insets), while, in vitro, rosiglitazone only induced differentiation of liposarcoma cells (data not shown). By using immunofluorescent double staining for vessels (MECA-32) and proliferation (PCNA), we found a significant decrease in endothelial cell proliferation in rosiglitazone-treated U87 (47\%) and LLC (44\%) as compared with controls (Figure 6e).

To exclude the possibility that the subcutaneous tumor location accounted for sensitivity to antiangiogenic therapy with TZDs, we used an orthotopic human prostate cancer model (24). The prostate cell line (LNCaP) weakly expresses PPAR $\gamma$, is relatively resistant to TZDs in vitro, and produces PSA (9). We treated established orthotopic prostate tumors with a serum PSA between 40 and $60 \mathrm{ng} / \mathrm{ml}$. After 17 days of treatment with rosiglitazone $(100 \mathrm{mg} / \mathrm{kg} / \mathrm{d})$ or troglitazone $(200 \mathrm{mg} / \mathrm{kg} / \mathrm{d})$, PSAs were $73 \pm 37 \mathrm{ng} / \mathrm{ml}$ or $100 \pm 45 \mathrm{ng} / \mathrm{ml}$, respectively, compared with the control group of $235 \pm 47 \mathrm{ng} / \mathrm{ml}$. Because TZDs can decrease PSA produced by tumor (LNCaP) cells in vitro with minimal inhibition of tumor cell proliferation (9), we excised tumors to determine whether TZDs had an effect on orthotopic tumor growth. Rosiglitazone- and troglitazone-treated tumors were $72 \%$ and $52 \%$ smaller by weight than control tumors, respectively. In addition, TZD-treated tumors showed a significant decrease in vessel density (data not shown). Therefore, the antitumor effect of PPAR $\gamma$ ligands can be mediated by antiangiogenesis in both subcutaneous and orthotopic tumor locations.

\section{Systemic therapy with PPAR $\gamma$ ligands prevents} metastatic invasion

We next studied the effect of TZDs on tumor metastasis. Mice were treated for 17 days with rosiglitazone (100 $\mathrm{mg} / \mathrm{kg} / \mathrm{d}$ ) or vehicle (methylcellulose) after removal of primary LLC. Removal of the primary LLC decreases the circulating angiogenesis inhibitor angiostatin, resulting in rapid growth of lung metastases (23). In mice treated with vehicle, there was marked growth of lung metastases (Figure 7a). The normal lung tissue was almost 


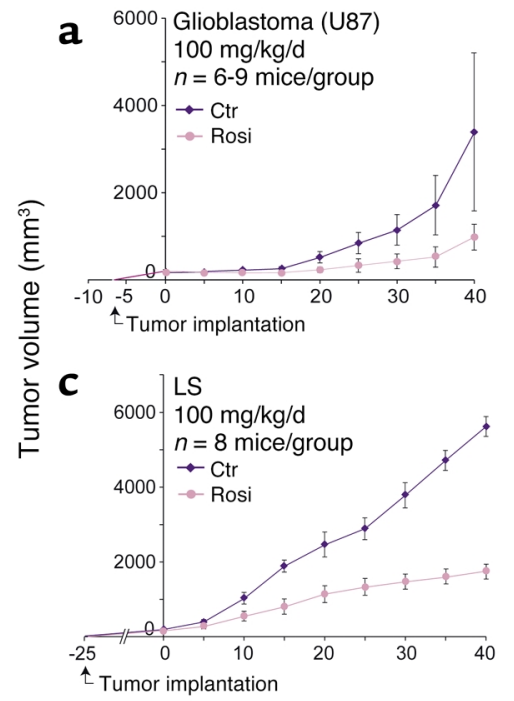

Treatment day

completely replaced by growing invasive metastases. The lung weights in these mice were $481 \pm 85 \mathrm{mg}$ (Figure 7b). In contrast, mice treated with rosiglitazone at 50 and $100 \mathrm{mg} / \mathrm{kg} / \mathrm{d}$ showed no established invasive metastases (Figure 7a), with lung weights of $194 \pm 22$ and $165 \pm 13$ $\mathrm{mg}$, respectively (Figure $7 \mathrm{~b}$ ), versus normal lung weights of $161 \pm 8 \mathrm{mg}$ (Figure 7b).

Individual LLC tumor cells in the rosiglitazone-treated mice were found inside the blood vessels, as demonstrated by hematoxylin-and-eosin staining (Figure 7c).

\section{Figure 5}

Systemic therapy with PPAR $\gamma$ ligands inhibits primary tumor growth. After tumors were $100-200 \mathrm{~mm}^{3}$ in size, rosiglitazone treatment $(100 \mathrm{mg} / \mathrm{kg} / \mathrm{d})$ was initiated (day 0$)$. On the last day of treatment, the statistical difference between control (Ctr; blue) and treated (pink) groups was determined by Student's $t$ test. (a) Glioblastoma (U87) $(P<0.01)$. (b) LLC $(P<0.001)$. (c) Liposarcoma (LS) $(P<0.001)$. (d) Rhabdomyosarcoma (RMS) $(P<0.001)$.

To confirm that these cells were tumor cells, we performed immunofluorescence staining for pericentrin, a centrosomal protein used to detect neoplastic cells (34). LLC tumor cells in control lungs with invasive metastasis show a high level of centrosome abnormalities (>50\%), as demonstrated by immunofluorescence staining for pericentrin (Figure 7, $\mathrm{d}$ and e, insets). In rosiglitazone-treated mice, tumor cells with centrosome abnormalities were only located inside PECAM-stained lung blood vessels (Figure 7, d and e). No LLC tumor cells were detected outside the vessels. These results suggest that rosiglitazone inhibits metastasis by preventing invasion of tumor cells through the endothelium.

It is widely appreciated that matrix metalloproteinases (MMPs) can promote tumor cell invasion while TIMPs decrease invasion. In order to determine whether rosiglitazone had an effect on tumor-associated MMP activity,
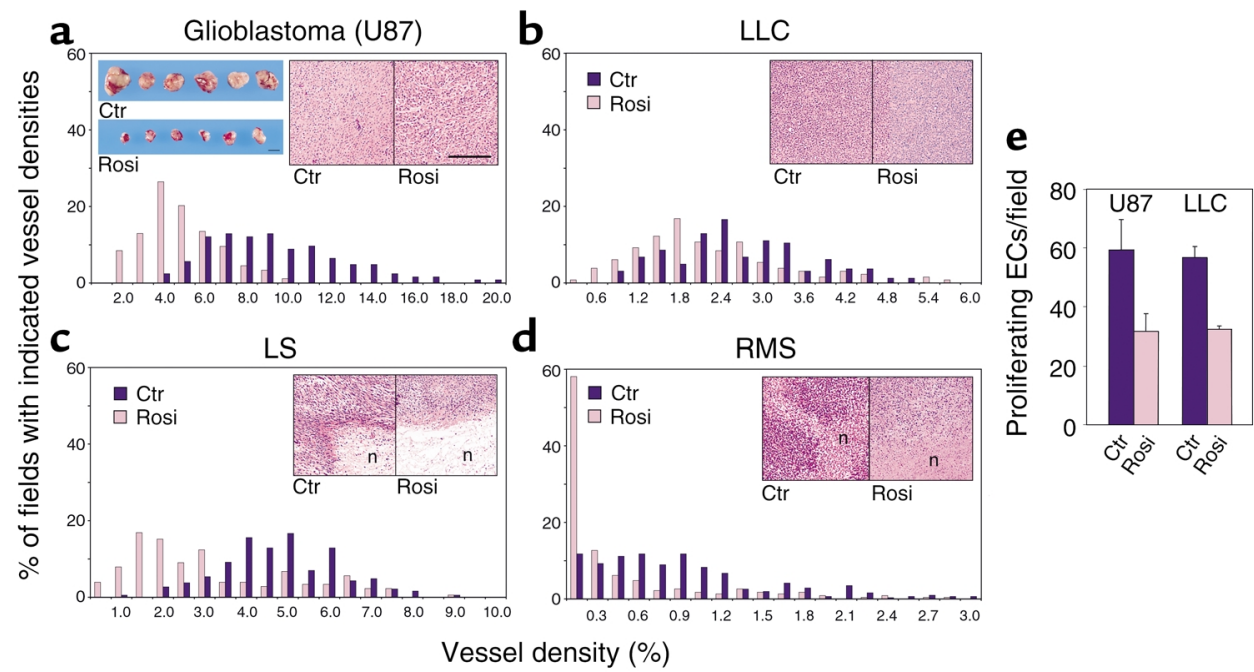

\section{Figure 6}

PPAR $\gamma$ ligand therapy reduces vessel density and endothelial cell proliferation. (a, blue inset) Representative control and treated glioblastoma tumors on treatment day 40 are shown. Scale bar, $1 \mathrm{~cm}$. (a-d, insets) Hematoxylin-and-eosin staining of tumors from control and rosiglitazone-treated mice show no signs of cytologic differentiation. In both liposarcoma and rhabdomyosarcoma, increased necrosis ( $\mathrm{n}$ ) was observed in treated tumors. Scale bar, $200 \mu \mathrm{m}$. (a-d) In each panel the $x$ axis represents vessel density defined as the percentage of vessel area (CD31)/tumor area in each tumor field. The $y$ axis indicates the percent of analyzed fields with the given vessel density. Bars further to the right represent fields with higher microvessel density. Upon rosiglitazone treatment, a significant decrease in vessel density was observed for all tumors as indicated by left-shifting of all histograms $(P<0.001)$. For example, in rosiglitazone-treated glioblastoma, $4 \%$ vessel density is present in $28 \%$ of all counted fields. Total fields scored per tumor (Ctr:Rosi): U87, 124:178; LLC, 163:131; LS, 186:178; RMS, 314:229. (e) Endothelial cell (EC) proliferation in rosiglitazone-treated and control U87 and LLC tumors as determined by immunofluorescent double staining (MECA-32 and PCNA). U87, $P<0.001$; LLC, $P<0.05$. Total fields scored per tumor (Ctr:Rosi): LLC, 36:26; U87, 34:33. 


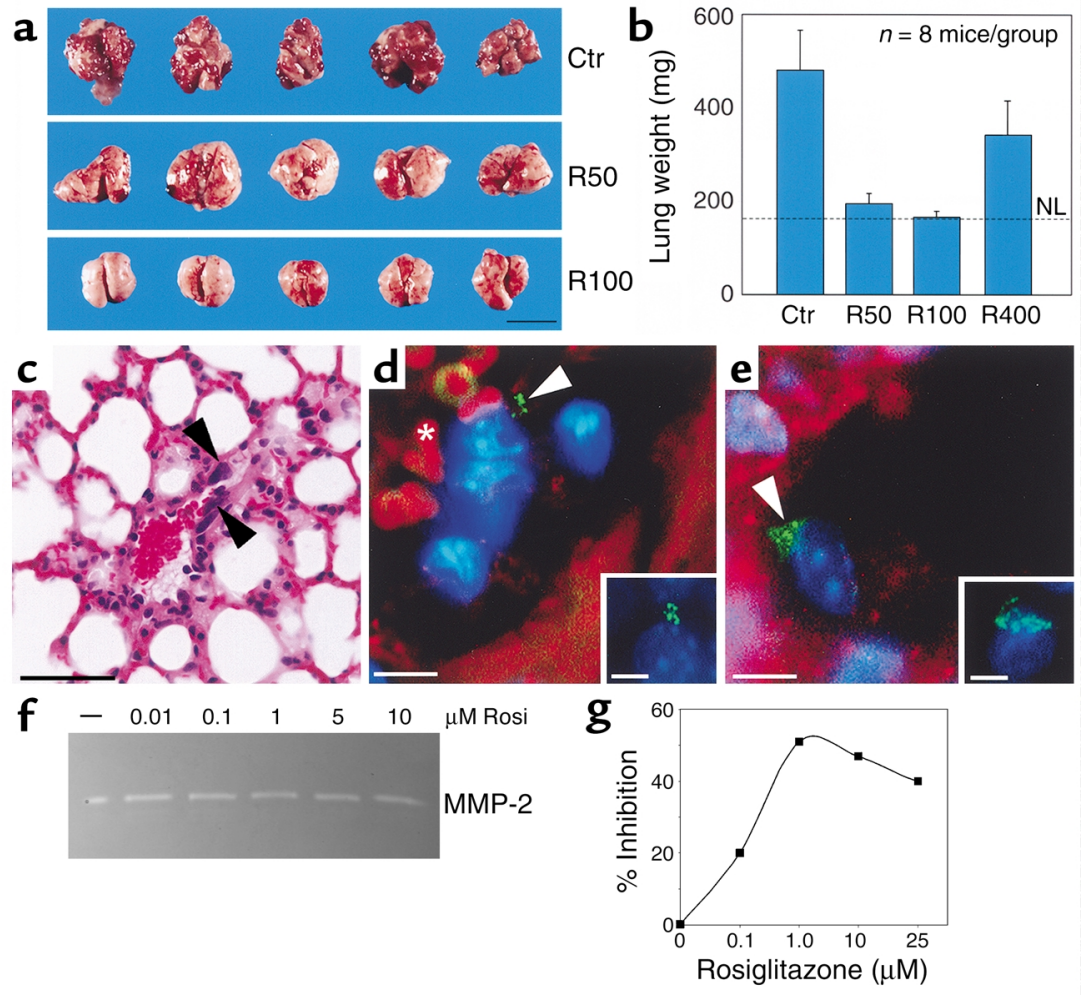

Figure 7

Systemic therapy with PPAR $\gamma$ ligands prevents metastatic invasion after removal of primary LLC. (a) Visual comparison of mouse lungs on treatment day 17 with vehicle (Ctr) or rosiglitazone (R50, $50 \mathrm{mg} / \mathrm{kg} / \mathrm{d}$; R100, $100 \mathrm{mg} / \mathrm{kg} / \mathrm{d}$ ). Scale bar, $1 \mathrm{~cm}$. (b) Rosiglitazone (R50 and R100) prevents metastasis, as represented by a significant decrease in lung weight, which correlates with tumor burden. R400, $400 \mathrm{mg} / \mathrm{kg} / \mathrm{d}$. NL, normal lung weight. (c) Hematoxylin-and-eosin staining showing individual LLC tumor cells inside blood vessels (black arrowheads) in lungs of rosiglitazone-treated mice. Scale bar, $50 \mu \mathrm{m}$. (d and e, insets) Typical patterns of centrosomal abnormalities in control LLC metastasis, as demonstrated by immunofluorescence staining for pericentrin (green). ( $\mathbf{d}$ and $\mathbf{e}$ ) In rosiglitazonetreated mouse lungs, immunofluorescence double staining for pericentrin and PECAM (endothelial marker) demonstrates LLC tumor cells inside blood vessels. Abnormal centrosomes of tumor cells stained with pericentrin are green (white arrowheads), and endothelium is red. The asterisk shows red blood cells ( $\mathbf{d}$, red). Hoechst staining of nuclei is blue. Scale bar, $5 \mu \mathrm{m}$. ( $\mathbf{f}$ and $\mathbf{g}$ ) Effect of rosiglitazone treatment on MMP and TIMP activity. Representative gelatin zymograms demonstrate that the activity of the predominant gelatinase detected in HUVECs appears to be unaffected by rosiglitazone treatment (f). In contrast, TIMP bioactivity assays demonstrate an increase in TIMP activity up to approximately $1.0 \mu \mathrm{M}$ rosiglitazone $(\mathbf{g})$.

we tested the conditioned media of endothelial cells that were treated with rosiglitazone via gelatin zymography. The predominant gelatinase detected was MMP-2 (gelatinase A), the activity of which appeared to be unaffected by rosiglitazone treatment (Figure 7f). Given that the net proteolytic activity is a function of the balance between MMPs and their endogenous inhibitors, the TIMPs, we next assayed these same samples in a radiometric enzyme assay for MMP inhibitory activity $(25,26)$. We found that rosiglitazone treatment resulted in an increase in MMP inhibitory bioactivity against the backdrop of approximately the same gelatinase activity (Figure $7 \mathrm{~g}$ ). Interestingly, this inhibitory profile exhibited the same biphasic response noted for the inhibition of endothelial cell proliferation and corneal neovascularization.

\section{Discussion}

The role of PPAR $\gamma$ in tumors has been widely studied. PPAR $\gamma$ ligands have been reported to have direct effects on tumor cells. We show that, by inhibiting angiogenesis, PPAR $\gamma$ ligands may have clinical application in treating not only primary tumor growth but metastatic growth as well, independent of tumor cell PPAR $\gamma$ expression or direct TZD-induced inhibition of tumor cell proliferation.

Our proliferation studies show that endothelial and tumor cells display markedly different sensitivities to low doses of TZDs in vitro. The antiproliferative endothelial cell effects after 72 hours of treatment with $1-10 \mu \mathrm{M}$ rosiglitazone are similar to those reported in HUVECs and choroidal endothelial cells $(15,18)$. Furthermore, at lower concentrations $(0.01-0.1 \mu \mathrm{M})$ of rosiglitazone that correspond to the range of its affinity for binding PPAR $\gamma$ (30), we observed similar endothelial cell inhibition after 7 days. These concentrations also activate PPAR $\gamma$ in fibroblasts and adipocytes (27). Most PPAR $\gamma$ activation markers have been identified in adipocytes, such as adipocyte fatty acid-binding protein (aP2) (35); to date, none have been described for the endothelial cell system. Consequently, we used ligand-induced PPAR $\gamma$ degradation as a surrogate marker of activation. These results indicate that PPAR $\gamma$ is activated by its ligands in endothelial cells and suggest that the inhibition of proliferation of endothelial cells by TZDs may be a direct result of this activation. Importantly, the levels of rosiglitazone that inhibit endothelial proliferation are readily achieved in patients undergoing standard antidiabetic rosiglitazone treatment (36).

TZDs have been shown to inhibit tumor cell proliferation in a PPAR $\gamma$-independent manner. In PPAR $\gamma$-deficient embryonic stem cells, TZDs $(25 \mu \mathrm{M})$ inhibit translation initiation by phosphorylation of eIF- $2 \alpha$ (12). Rosiglitazone concentrations of $5 \mu \mathrm{M}$ and higher led to phosphorylation of eIF- $2 \alpha$ in HUVECs, suggesting that inhibition of proliferation of endothelial cells was mediated by a PPAR $\gamma$-independent pathway. However, we observed that $0.01-1 \mu \mathrm{M}$ of rosiglitazone, the concentration range at which PPAR $\gamma$ was activated, had the strongest antiproliferative effect on endothelial cells in vitro. Therefore, the inhibitory activity of 
rosiglitazone on endothelial cells is most likely mediated through PPAR $\gamma$, whereas PPAR $\gamma$-independent effects on translation may become important when higher concentrations of rosiglitazone are used.

In addition to direct action on endothelium, tumor angiogenesis can also be affected by indirect mechanisms. Our results showed that TZDs decreased VEGF production by tumor cells. Antiangiogenesis can result from a decrease of stimulators (e.g., VEGF and bFGF) and/or an increase of inhibitors (e.g., thrombospondin) in the tissue. It has been shown that PPAR $\gamma$ activation downregulates leptin and TNF- $\alpha$, both of which are angiogenic factors $(30,37)$. Other reports show that PPAR $\gamma$ activation upregulates the expression of the angiogenesis inhibitor maspin and CD36, the receptor for antiangiogenic thrombospondin $(5,38,39)$.

Antiangiogenic activity can also be mediated indirectly by pericytes and macrophages, which both express PPAR $\gamma$. Pericytes (also known as mural cells) have recently been shown to be present in significant numbers in tumor blood vessels (40). Therefore, PPAR $\gamma$ ligands, which inhibit vascular smooth muscle cell proliferation and migration (30), may indirectly affect endothelial cell survival. Our CAM assay results suggest that PPAR $\gamma$ ligands have macrophage-independent effects in vivo. However, PPAR $\gamma$ ligands have been shown to inhibit macrophage activation and invasion, which are important modulators of tumor angiogenesis $(30,41-43)$. Thus, PPAR $\gamma$ ligands may inhibit tumor angiogenesis by indirect as well as by direct mechanisms.

Surprisingly, higher doses of rosiglitazone (e.g., 400 $\mathrm{mg} / \mathrm{kg} / \mathrm{d}$ ) were less antiangiogenic than lower doses (e.g., $50 \mathrm{mg} / \mathrm{kg} / \mathrm{d}$ ). Such a biphasic effect is not unprecedented, because other angiogenesis inhibitors, such as IFN- $\alpha$, have less antiangiogenic activity at higher doses (20). This biphasic response is indicative of a negative feedback loop that operates at higher doses. One possibility is the drug-induced degradation of PPAR $\gamma$, which at high dose might lead to complete depletion, and thus unresponsiveness. Alternatively, higher doses of rosiglitazone might induce enzymatic drug metabolism pathways. The results from twicedaily administration suggest that the doses of TZDs may be lowered further to achieve efficacy similar to that achieved with daily administration. Such a result is consistent with reports that frequent low doses of chemotherapy have increased antiangiogenic efficacy and that continuous administration of angiostatin increases tumor suppression at significantly reduced doses $(29,44,45)$. This is presumably because endothelial inhibition is more related to duration of drug exposure than to peak levels achieved.

Several cancer cell lines, including bladder, breast, and thyroid carcinoma, require higher doses $(50-100 \mu \mathrm{M})$ for inhibition of proliferation or are relatively resistant to antiproliferative effects of TZDs in vitro $(5,8,10,11$, 46). Our tumor lines were minimally inhibited by rosiglitazone in vitro but were dramatically suppressed in vivo, in agreement with reports that TZDs have anti- tumor effects in PPAR $\gamma$-negative tumors (12). Our antitumor effect resulted from a reduction in tumor microvessel density and a decrease in endothelial cell proliferation. Importantly, no signs of tumor cell differentiation or reduction in tumor cell proliferation were observed in treated tumors. This, together with the direct effects on the endothelium, suggests that the observed antitumor activity of TZDs is not solely mediated by a cell-autonomous response of the tumor cells.

In our studies, systemic PPAR $\gamma$ ligand therapy prevented metastatic invasion of LLC, consistent with the report that TZD administration inhibited the metastatic spread of thyroid tumors to the liver (11). Vascular invasion involves ECM proteolysis. Moreover, endothelial and tumor cells secrete proteinase inhibitors, such as TIMPs or plasminogen activator inhibitors (PAIs), which suppress tumor invasion and angiogenesis (26, 47). Our studies demonstrate that rosiglitazone increases the inhibition of MMP activity in HUVECs, and others have shown that PPAR $\gamma$ ligands can increase PAI-1 expression in human endothelial cells $(14,15)$. The mechanism by which PPAR $\gamma$ ligands such as rosiglitazone may prevent the establishment and progression of metastatic disease includes the previously described antiangiogenic effects such as inhibition of endothelial proliferation and decrease in VEGF secretion, in addition to the upregulation of negative regulators of metastatic invasion such as MMP inhibitors.

Our studies suggest that PPAR $\gamma$ ligands may be useful in treating angiogenic diseases such as cancer because of their effect on endothelium. Moreover, the endothelium has become an important target in the treatment of non-neoplastic diseases such as psoriasis, endometriosis, and arthritis. PPAR $\gamma$ ligands may represent a novel antiangiogenic therapy in rheumatoid arthritis (48). Furthermore, it is now becoming evident that multi-drug-resistant tumors can be effectively targeted by antiangiogenic chemotherapy (also called metronomic) chemotherapy $(29,45)$. As an orally administered FDA-approved drug used in over 2 million people with minimal side effects, rosiglitazone would be ideally suited for use in combination with other antiangiogenesis regimes and holds great promise to complement conventional modalities for cancer treatment.

\section{Acknowledgments}

We thank Carmen Barnes, Diane Bielenberg, Lynn Chang, Michael Fannon, Sui Huang, Mark Kieran, and Wei Wen for suggestions in preparing the manuscript. The excellent technical assistance of Geraldine Jackson, Andrea Laforme, Marty Olesiak, and Ricky Sanchez is acknowledged. We thank Kristin Gullage for photography. This study was supported by a sponsored research grant to Children's Hospital from Entremed Inc. (Rockville, Maryland, USA). This work was supported by a European Molecular Biology Organization fellowship and by the Kopf Family Foundation (both to A. Kaipainen) and by NIH grant T32CA09535 (to D. Panigrahy). 
1. Rosen, E.D., and Spiegelman, B.M. 2001. PPAR $\gamma$ : a nuclear regulator of metabolism, differentiation, and cell growth. J. Biol. Chem. 276:37731-37734.

2. Barak, Y., et al. 1999. PPAR- $\gamma$ is required for placental, cardiac, and adipose tissue development. Mol. Cell. 4:585-595.

3. Tontonoz, P., Hu, E., and Spiegelman, B.M. 1994. Stimulation of adipogenesis in fibroblasts by PPAR $\gamma 2$, a lipid activated transcription factor. Cell. 79:1147-1156.

4. Tontonoz, P., Nagy, L., Alvarez, J.G., Thomazy, V.A., and Evans, R.M. 1998. PPAR $\gamma$ promotes monocyte/macrophage differentiation and uptake of oxidized LDL. Cell. 93:241-252.

5. Mueller, E., et al. 1998. Terminal differentiation of human breast cancer through PPAR $\gamma$. Mol. Cell. 1:465-470.

6. Sarraf, P., et al. 1998. Differentiation and reversal of malignant changes in colon cancer through PPAR $\gamma$. Nat. Med. 4:1046-1052.

7. Tontonoz, P., et al. 1991. Terminal differentiation of human liposarcoma cells induced by ligands for peroxisome proliferator-activated receptor $\gamma$ and the retinoid X receptor. Proc. Natl. Acad. Sci. USA. 94:237-241.

8. Elstner, E., et al. 1998. Ligands for peroxisome proliferator-activated receptor $\gamma$ and retinoic acid and receptor inhibit growth and induce apoptosis of human breast cancer cells in vitro and in BNX mice. Proc. Natl. Acad. Sci. USA. 95:8806-8811.

9. Kubota, T., et al. 1998. Ligand for peroxisome-activated receptor $\gamma$ (Troglitazone) has potent antitumor effect against human prostate cancer both in vivo and in vitro. Cancer Res. 58:3344-3352.

10. Nakashiro, K., et al. 2001. Role of peroxisome proliferator-activated receptor $\gamma$ and its ligands in non-neoplastic and neoplastic human urothelial cells. Am. J. Pathol. 159:591-597.

11. Ohta, K., Endo, T., Haraguchi, K., Hershman, J.M., and Onaya, T. 2001 Ligands for peroxisome proliferator-activated receptor $\gamma$ inhibit growth and induce apoptosis of human papillary thyroid carcinoma cells. J. Clin. Endocrinol. Metab. 86:2170-2177.

12. Palakurthi, S.S., Aktas, H., Grubissich, L.M., Mortensen, R.M., and Halperin, J.A. 2001. Anticancer effects of thiazolidinediones are independent of peroxisome proliferator-activated receptor $\gamma$ and mediated by inhibition of translation initiation. Cancer Res. 61:6213-6218.

13. Folkman, J. 2000. Tumor angiogenesis. In Cancer medicine. 5th edition. J.F. Holland et al., editors. B.C. Decker Inc. Hamilton, Ontario, Canada. $132-152$.

14. Marx, N., Bourcier, T., Sukhova, G.K., Libby, P., and Plutzky, J. 1999. PPAR $\gamma$ activation in human endothelial cells increases plasminogen activator inhibitor type-1 expression: PPAR $\gamma$ as a potential mediator in vascular disease. Arterioscler. Thromb. Vasc. Biol. 19:546-551.

15. Xin, X., Yang, S., Kowalski, J., and Gerritsen, M.E. 1999. Peroxisome proliferator-activated receptor $\gamma$ ligands are potent inhibitors of angiogenesis in vitro and in vivo. J. Biol. Chem. 274:9116-9121.

16. Bishop-Bailey, D., and Hla, T. 1999. Endothelial cell apoptosis induced by the peroxisome proliferator-activated receptor (PPAR) ligand 15-deoxy-prostaglandin $\mathrm{J}_{2}$. J. Biol. Chem. 274:17042-17048.

17. Gralinski, M.R., Rowse, P.E., and Breider, M.A. 1998. Effects of troglitazone and pioglitazone on cytokine-mediated endothelial cell proliferation in vitro. J. Cardiovasc. Pharmacol. 31:909-913.

18. Murata, T., et al. 2000. Peroxisome proliferator-activated receptor- $\gamma$ ligands inhibit choroidal neovascularization. Invest. Ophthalmol. Vis. Sci. 41:2309-2317.

19. Inoue, K., et al. 2001. Expression of peroxisome proliferator-activated receptor (PPAR)-gamma in human lung cancer. Anticancer Res. 21:2471-2476

20. Slaton, J.W., Perrotte, P., Inoue, K., Dinney, C., and Fidler, I.J. 1999. Interferon- $\alpha$-mediated down-regulation of angiogenesis-related genes and therapy of bladder cancer are dependent on optimization of biological dose and schedule. Clin. Cancer Res. 5:2726-2734.

21. D’Amato, R., Loughnan, M.S., Flynn, E., and Folkman, J. 1994. Thalidomide is an inhibitor of angiogenesis. Proc. Natl. Acad. Sci. USA. 91:4082-4085.

22. Masferrer, J.L., et al. 2000. Antiangiogenic and antitumor activities of cyclooxgenase-2 inhibitors. Cancer Res. 60:1306-1311.

23. O'Reilly, M.S., et al. 1994. Angiostatin: a novel angiogenesis inhibitor that mediates the suppression of metastases by a lewis lung carcinoma. Cell. 79:315-328.

24. Stephenson, R.A., et al. 1992. Metastatic model for human prostate cancer using orthotopic implantation in nude mice. J. Natl. Cancer Inst. 84:951-957.

25. Braunhut, S., and Moses, M. 1994. Retinoids modulate endothelial cell production of matrix-degrading proteases and tissue inhibitors of metalloproteinases (TIMP). J. Biol. Chem. 269:13472-13479.

26. Moses, M.A., Sudhalter, J., and Langer, R. 1990. Identification of an inhibitor of neovascularization from cartilage. Science. 248:1408-1410.

27. Hauser, S., et al. 2000. Degradation of the peroxisome proliferator-activated receptor $\gamma$ is linked to ligand dependent activation. J. Biol. Chem. 275:18527-18533.

28. Moses, M.A., et al. 1999. Troponin I is present in human cartilage and inhibits angiogenesis. Proc. Natl. Acad. Sci. USA. 96:2645-2650.

29. Browder, T., et al. 2000. Antiangiogenic scheduling of chemotherapy improves efficacy against experimental drug-resistant cancer. Cancer Res. 60:1878-1886

30. Berger, J., and Moller, D.E. 2002. Mechanism of action of PPARs. Annu. Rev. Med. 53:409-435.

31. Ribatti, D., Vacca, A., Roncali, L., and Dammacco, F. 2000. The chick embryo chorioallantoic membrane as a model for in vivo research on antiangiogenesis. Curr. Pharm. Biotechnol. 1:73-82.

32. Folkman, J. 2001. Angiogenesis-dependent diseases. Semin. Oncol. 28:536-542.

33. Hlatky, L., Hahnfeldt, P., and Folkman, J. 2002. Clinical application of antiangiogenic therapy: microvessel density, what it does and does not tell us. J. Natl. Cancer Inst. 94:883-893.

34. Pihan, G.A., et al. 1998. Centrosome defects and genetic instability in malignant tumors. Cancer Res. 58:3974-3985.

35. Elbrecht, A., et al. 1996. Molecular cloning, expression and characterization of human peroxisome proliferator activated receptors $\gamma 1$ and $\gamma 2$. Biochem. Biophys. Res. Commun. 224:431-437.

36. Freed, M.I., Allen, A., Jorkasky, D.K., and DiCicco, R.A. 1999. Systemic exposure to rosiglitazone is unaltered by food. Eur. J. Clin. Pharmacol. 55:53-56.

37. Sietta-Honigmann, M.R., et al. 1998. Biological action of leptin as an angiogenic factor. Science. 281:1683-1686.

38. Zhang, M., Volpert, O., Shi, Y.H., and Bouck, N. 2000. Maspin is an angiogenesis inhibitor. Nat. Med. 6:196-199.

39. Jimenez, B., et al. 2000. Signals leading to apoptosis-dependent inhibition of neovascularization by thrombospondin-1. Nat. Med. 6:41-48.

40. Morikawa, S., et al. 2002. Abnormalities in pericytes on blood vessels and endothelial sprouts in tumors. Am. J. Pathol. 160:985-1000.

41. Sunderkotter, C., Steinbrink, K., Goebeler, M., Bhardwaj, R., and Sorg, C. 1994. Macrophages and angiogenesis. J. Leukoc. Biol. 55:410-422.

42. Ricote, M., Li, A.C., Willson, T.M., Kelly, C.J., and Glass, C.K. 1998. The peroxisome proliferator-activated receptor- $\gamma$ is a negative regulator of macrophage activation. Nature. 391:79-82.

43. Pasceri, V., Wu, H.D., Willerson, J.T., and Yeh, E.T.H. 2000. Modulation of vascular inflammation in vitro and in vivo by peroxisome proliferator-activated receptor- $\gamma$ activators. Circulation. 101:235-238.

44. Drixler, T.A., et al. 2000. Continuous administration of angiostatin inhibits accelerated growth of colorectal liver metastases after partial hepatectomy. Cancer Res. 60:1761-1765.

45. Klement, G., et al. 2000. Continuous low-dose therapy with vinblastine and VEGF receptor-2 antibody induces sustained tumor regression without overt toxicity. J. Clin. Invest. 105:15-24.

46. Clay, C.E., et al. 2001. Magnitude of peroxisome proliferator-activated receptor-gamma activation is associated with important and seemingly opposite biological responses in breast cancer cells. J. Investig. Med. 49:413-420.

47. Liotta, L.A., Steeg, P.S., and Stetler-Stevenson, W.G. 1991. Cancer metastasis and angiogenesis: an imbalance of positive and negative regulation. Cell. 64:327-336.

48. Fahmi, H., Pelletier, J.P., and Martel-Pelletier, J. 2002. PPAR $\gamma$ ligands as modulators of inflammatory and catabolic responses in arthritis. An overview. J. Rheumatol. 29:3-14. 